\title{
Rôle des lacs-réservoirs amont : les grands lacs de Seine
}

\author{
par Gérard Villion \\ Institution Interdépartementale des Barrages-Réservoirs du Bassin de la Seine
}

\section{I $\square$ APERÇU HISTORIQUE DES AMÉNAGEMENTS}

\subsection{Historique des protections}

Jusqu'au début du siècle, la lutte contre les inondations a fait appel à des travaux de protections et d'aménagements locaux. A partir du Directoire, ceux-ci ont été structurés en vue d'une défense longitudinale et se sont poursuivis jusqu'au milieu du XIXème siècle.

Parallèlement, ce siècle a connu l'essor de grands projets de protection de la capitale. MM. Poirée (1824 puis 1839), Chanoine (après 1840), Belgrand (1846), Cambuzat (1860), Desfontaines (1857) et Mary (1868) avancent l'idée de création de tels réservoirs soit en plaine (la loi du 28 mai 1858 instituait des servitudes tendant au maintien des champs de submersion dans les vallées de la Seine et de la Marne), soit en "montagne" (stockage de $100 \mathrm{Mm}^{3}$ - millions de $\mathrm{m}^{3}$ - en 12 réservoirs dans le Morvan dont l'exemple est donné par le barrage des Settons, de $23 \mathrm{Mm}^{3}$, inauguré en 1858) et la réalisation de dérivations contournant Paris, projets déjà esquissés au XVIIème siècle.

La Seine en 1910, avec un débit de l'ordre de $2400 \mathrm{~m}^{3} / \mathrm{s}$, a débordé par dessus les parapets de la capitale après avoir inondé de nombreuses agglomérations de la banlieue. C'est à la suite de cette crue que les idées émises au XIXème siècle, ont commencé à trouver un écho favorable.

\subsection{La commission PICARD}

Suite à la crue, le décret du 9 février 1910 met en place la commission Picard qui sera chargée de rechercher les causes des inondations, d'étudier et de proposer les moyens de les empêcher ou, tout au moins, d'en diminuer l'intensité. Concernant les travaux susceptibles de réduire les crues dans le bassin de la Seine, notamment à Paris et sa banlieue, une sous-commission est créée dont le rapport est inspiré principalement par l'Ingénieur Drogue.

La sous-commission puis la commission, examinent les possibilités d'action par des réservoirs à buts multiples. En 1910, 13 réservoirs représentant une capacité de $80 \mathrm{Mm}^{3}$ existent et sont principalement utilisés pour les besoins de la navigation. Au 15 janvier 1910, ils contiennent $68 \mathrm{Mm}^{3}$ et ne laissent donc qu'une disponibilité inférieure à $12 \mathrm{Mm}^{3}$ face aux milliards de $\mathrm{m}^{3}$ débités par la Seine. Les conclusions de la commission sont que les réservoirs de montagne représentent des ouvrages trop coûteux pour privilégier l'écrêtement des crues et ne disciplinent qu'un faible bassin versant et que les réservoirs de plaine mobilisent des surfaces importantes pour lesquelles l'étendue des dommages résultant de leur implantation n'est pas chiffrée. Ces arguments conduisent la commission à donner un avis défavorable à ces mesures au profit d'un important programme de défense locale consistant d'une part à l'ouverture d'un bras de décharge calibré à $500 \mathrm{~m}^{3} / \mathrm{s}$ entre Annet-sur-Marne et Epinay-sur-Seine et d'autre part, un approfondissement de la Seine entre Suresnes et Bougival.

The floods of 1910 and 1924 led to the equipment of the upper Seine Basin with large scale infrastructures, which include reservoirs with multiple uses. This presentation provides a cursory historical background, then provides a brief introduction to the Seine four "great lakes". These lakes have a capacity of $820 \mathrm{Mm}^{3}$ ( 830 million cubic metres or 29,311 million cubic feet), which can be used to lower the flood magnitudes. With the MSBR (Modele Seine barrages-Résenvoirs) - a dam-reservoir propagation model designed by Hydratec for the Seine great lakes - it is possible to simulate the impact these lakes would have had for the major floods of the past and, in real time, to quantify the effect of these reservoirs on the Marne and Seine under flooding conditions, and thus to predict the evolution in time and space of future floods in the lle de France region. These controling structures and their associated management have significantly contributed to flood reduction. Yet the flood-reducing role of the Seine great lakes remains a limited one, for two reasons : the large flood volumes involved relative to the reservoir capacities and the large distance of these lakes from the Paris region. 
Les travaux ponctuels de première urgence sont entrepris immédiatement, mais la guerre mondiale survenant, beaucoup de projets restent en l'état.

\subsection{Convergence des aspects crue et soutien d'étiage}

Parallèlement à la commission Picard, des études sont entreprises notamment celle commencée par M. Chabal en 1914 sur l'alimentation en eau de Paris. Elle aboutit, en 1920 , à la mise au point d'un projet grandiose de régularisation du débit de la Seine par la création d'un grand nombre de réservoirs disséminés dans tout le bassin. Ils devaient permettre la suppression des grandes crues, la récupération d'énergie hydraulique et la fourniture d'eau potable pour la région parisienne.

En effet, les aspects liés au soutien des étiages progressent à la suite des besoins rencontrés pendant la guerre : difficulté et crise des transports conduisant à valoriser la navigation, pénurie et cherté du charbon menant à l'énergie hydroélectrique et augmentation des besoins en eau potable pour une population totale de l'agglomération parisienne estimée par M. Chabal à près de 10 millions d'habitants en l'an 2000 .

Ces différents aspects revalorisent le projet de régularisation des rivières du bassin par la réalisation de réservoirs pour les besoins en eau potable face aux projets de recherche de nouvelles ressources provenant d'autres bassins.

M. Chabal propose ainsi en 1920 , se basant sur la ligne d'eau de la crue de 1910 qu'il envisage de rabaisser de $5 \mathrm{~m}$, d'emmagasiner $2400 \mathrm{Mm}^{3}$ répartis sur 23 réservoirs à réaliser en 2 phases : commencer par écrêter les crues à $1500 \mathrm{~m}^{3} / \mathrm{s}$ par un stockage de $1260 \mathrm{Mm}^{3}$ répartis sur 11 réservoirs, puis les réduire à $1225 \mathrm{~m}^{3} / \mathrm{s}$ par des retenues complémentaires de $1140 \mathrm{Mm}^{3}$ à l'aide de 12 réservoirs.

Ainsi, au début des années "20", les antagonismes créés pour la lutte contre les crues entre les projets Drogue et les réservoirs du projet Chabal se retrouvent dans ceux existant pour le soutien des étiages (projet Chabal face aux nouvelles ressources hors bassin).

En 1923, M. Béaud, Conseiller Général de la Seine, suggère l'aménagement de la Seine par des ouvrages de régulation ayant le double objectif : lutte contre les crues et alimentation en eau potable.

\subsection{La commission DUSUZEAU}

En janvier 1924, une nouvelle crue cause d'importants dommages en banlieue parisienne, alors que les dégâts intramuros sont limités grâce aux travaux réalisés depuis 1910 . Une nouvelle commission d'études, présidée par l'Inspecteur Général Dusuzeau, recommande dans son rapport du 11 décembre 1925 , la construction en première urgence de barrages-réservoirs qui assureraient d'une part des prélèvements en crues et d'autre part une production hydroélectrique et une contribution à l'alimentation en eau.

Pour approuver ces mesures, la commission s'appuie sur les considérations suivantes :

"on ne saurait penser à réaliser, pour une capacité donnée à l'amont, un abaissement défini à Paris",

"la défense ne peut être obtenue à Paris par le seul moyen de réservoirs",

"il est intéressant d'envisager la création progressive de réservoirs dans des buts définis et spécialisés (par exemple, soutenir le débit d'étiage, produire de l'électricité, ou contribuer à l'alimentation des canaux) et de les utiliser aussi pour agir sur le niveau des crues".
Le programme de barrages d'une capacité de stockage de $1000 \mathrm{Mm}^{3}$ est approuvé par le Ministre des Travaux Publics le 14 janvier 1926 et le Service des Barrages-Réservoirs du Département de la Seine est créé, en 1928 pour la construction de ces ouvrages.

\subsection{Le programme de 1ère urgence}

Les premières réalisations comprennent 4 ouvrages :

- le barrage du Crescent sur la Cure, mis en service en 1931;

- le barrage du Bois-de-Chaumeçon sur le Chalaux, affluent de la Cure, en 1934.

Ces 2 premiers ouvrages sont réalisés par des producteurs d'électricité indépendants auxquels le Département de la Seine apporte des subventions en contre-partie d'engagements de mise à disposition de $24 \mathrm{Mm}^{3}$ d'eau pour la régularisation.

- le barrage-réservoir de Champaubert-aux-Bois $\left(23 \mathrm{Mm}^{3}\right)$ sur la Blaise, affluent de la Marne, en 1938 ;

- le barrage de Pannecière-Chaumard sur l'Yonne, commencé en 1939 et interrompu pendant la guerre.

$\mathrm{La}$ justification de chaque ouvrage diffère sensiblement. Les impératifs de rentabilité économique de l'époque exigeaient d'utiliser les ouvrages à des fins multiples et notamment pour l'hydroélectricité. Le barrage-réservoir de Pannecière-Chaumard, grâce à sa grande hauteur de chute, est ainsi équipé d'une usine hydroélectrique.

Le réservoir de Champaubert-aux-Bois n'a pu, en raison de la modicité des débits de lâchures et de sa faible hauteur de chute, être équipé d'une usine hydroélectrique mais sa justification est autre : il constitue, en fait, le modèle réduit des grands réservoirs de Champagne, préconisés en 2ème phase par la commission.

Ainsi, le premier programme représente la construction d'ouvrages en vallée principalement dans le Morvan. La crue de 1955 donne un second souffle à l'équipement du bassin amont avec la réalisation, en Champagne Humide, des lacs "Seine", "Marne" et "Aube", en dérivation des rivières du même nom.

\section{II $\square$ LE POTENTIEL DES GRANDS LACS DE SEINE}

\subsection{L'I.I.B.R.B.S.}

L'action entreprise par le Département de la Seine a été poursuivie par l'Institution Interdépartementale des BarragesRéservoirs du Bassin de la Seine (I.I.B.R.B.S.) créée en 1969 à la suite de la réforme administrative de la région parisienne. L'I.I.B.R.B.S. qui regroupe, les départements de Paris, des Hauts-de-Seine, de la Seine-Saint-Denis et du Valde-Marne, a pour charge d'exploiter les barrages-réservoirs existants et de poursuivre le programme de construction de nouveaux aménagements, en vue d'assurer la protection contre les inondations et la régularisation des débits d'étiage des rivières sur le territoire des collectivités qui la composent.

Evidemment, les réservoirs de l'Institution qui a pris récemment la dénomination "Les Grands Lacs de Seine", régularisent également les tronçons des rivières situées entre ces ouvrages et la région parisienne et concernent donc l'ensemble des habitants du bassin en aval de ces retenues. 
1. Carte du bassin versant de la Seine.

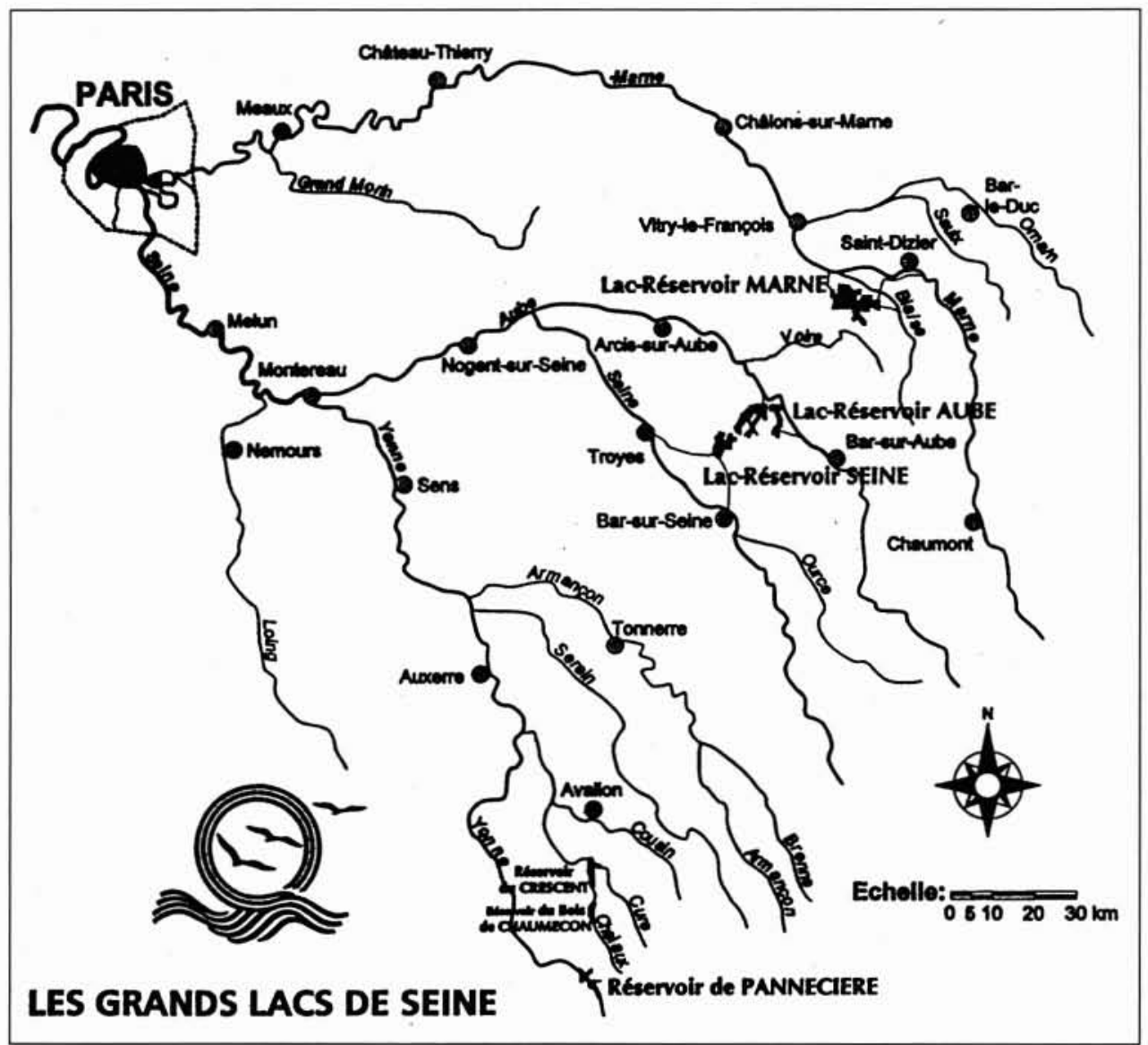

L'Institution possède 4 ouvrages localisés sur des terrains imperméables, soit sur les massifs granitiques du Morvan, soit sur les argiles du Gault de la Champagne Humide (fig 1).

\subsection{Le barrage-réservoir de Pannecière-Chaumard}

Cet ouvrage en béton, à voûtes multiples, est établi dans la haute vallée de l'Yonne. Sa capacité est de $80 \mathrm{Mm}^{3}$ pour un bassin versant contrôlé de $220 \mathrm{~km}^{2}$. Cet ouvrage, achevé après la guerre, a été mis en service en 1949.

\subsection{Le lac-réservoir "Seine" ou Lac de la Forêt d'Orient}

Ce réservoir, constitué de digues en terre, est établi en dérivation de la Seine en amont de Troyes. Il offre un volume de stockage de $205 \mathrm{Mm}^{3}$ pour un bassin versant contrôlé est de $2400 \mathrm{~km}^{2}$. Le canal d'amenée est susceptible de dériver $180 \mathrm{~m}^{3} / \mathrm{s}$, alors que le plus fort débit connu depuis 1888 est de l'ordre de $350 \mathrm{~m}^{3} / \mathrm{s}$ à Bar-sur-seine. Sa mise en service est intervenue en 1966.

\section{- 2.4 Le lac-réservoir "Marne" ou Lac du Der-Chantecoq}

Délimité également par des digues en terre, ce lac-réservoir est établi, au droit de Saint-Dizier, en dérivation de la Marne et de la Blaise, affluent de la Marne. Sa capacité est de $350 \mathrm{Mm}^{3}$ pour un bassin versant contrôlé est de $2950 \mathrm{~km}^{2}$. Les ouvrages d'amenée peuvent dériver jusqu'à $400 \mathrm{~m}^{3} / \mathrm{s}$, tandis que le plus fort débit observé en amont de l'ouvrage est un peu supérieur à $600 \mathrm{~m}^{3} / \mathrm{s}$. Cet ouvrage a été mis en service en 1974 et a englober l'ancien réservoir de Champaubert-aux-Bois.

\subsection{Le lac-réservoir "Aube" ou Lacs Aube}

Ce lac, constitué par deux bassins, lac Amance et lac Temple, fermés par des digues en terre, est implanté en dérivation de l'Aube près de Brienne-le-Château. Il représente une capacité de $170 \mathrm{Mm}^{3}$ pour un bassin versant contrôlé de $1700 \mathrm{~km}^{2}$. Sa prise d'eau en rivière permet de dériver $135 \mathrm{~m}^{3} / \mathrm{s}$ soit jusqu'aux $2 / 3$ du débit maximal connu en Aube sur ce site. Sa mise en service a commencé en 1989 et sa première mise en eau complète est intervenue en 1991.

\section{- 2.6 Le potentiel de l'Institution}

Ce sont ainsi environ $830 \mathrm{Mm}^{3}$ qui peuvent être stockés dans ces ouvrages, $820 \mathrm{Mm}^{3}$ pouvant être mobilisables pour l'écrêtement des crues et $800 \mathrm{Mm}^{3}$ pour le soutien des étiages suivant l'utilisation, en crue, des "tranches exceptionnelles" $\left(42 \mathrm{Mm}^{3}\right)$ et, en étiage, des "tranches de réserve" $\left(52 \mathrm{Mm}^{3}\right)$ de ces ouvrages.

L'Institution dispose également de $24 \mathrm{Mm}^{3}$, utilisés essentiellement pour l'étiage, des ouvrages du Crescent et du Bois-de-Chaumeçon gérés par Electricité de France et alimentés par un bassin versant de $520 \mathrm{~km}^{2}$.

\section{III — LES MODALITÉS D'EXPLOITATION DES GRANDS LACS DE SEINE}

Les modalités d'exploitation de chaque ouvrage sont détaillées dans un règlement d'eau, défini par un arrêté préfectoral des départements d'implantation, pris notamment après consultation des organismes de bassin et à l'issue d'une enquête publique dite "enquête hydraulique" qui porte sur 
l'ensemble des communes riveraines des rivières influencées par les effets de l'ouvrage.

Les objectifs d'écrêtement des crues et de soutien des étiages sont souvent apparus contradictoires comme en témoignent les études historiques précédemment citées. Pourtant, et l'exploitation des ouvrages existants le montre, des résultats sensibles sont obtenus concurremment sur ces deux plans.

En effet, alors que les étiages les plus durement ressentis des rivières du Bassin de la Seine ont lieu en été et en automne, les crues les plus dommageables apparaissent en hiver et au printemps ; celles d'hiver étant statistiquement les plus importantes.

Les règlements d'eau prévoient, dans ces conditions, un remplissage théorique progressif des barrages pendant l'hiver et le printemps et leur vidange pendant l'été et le début de l'automne avec des prolongements éventuels en fin d'automne (fig. 2).

Les creux disponibles pour l'écrêtement des crues sont donc décroissants de janvier à juin, tout en restant adaptés aux volumes statistiquement nécessaires à cet écrêtement. Dans ces conditions, à l'époque de l'année où les crues sont les plus dangereuses, on dispose de la capacité maximale de stockage, les réservoirs étant en fin de vidange ou en début de remplissage.

L'exploitation journalière est conduite en suivant une courbe d'objectifs de remplissage et de vidange en l'absence de crues ou d'étiages prononcés. Lorsqu'une crue importante survient, le débit total laissé en rivière à l'aval de l'ouvrage est limité à une valeur fixée dite "débit de référence". Le remplissage est donc majoré pendant les périodes de crues, et après la décrue le volume emmagasiné dans la retenue est supérieur au remplissage théorique. Des délestages sont alors effectués, en respectant le débit de référence, pour revenir progressivement dans une situation normale et reconstituer le potentiel d'intervention pour une éventuelle crue.

Les 4 ouvrages permettent ainsi de réaliser des prélèvements adaptés sur chacune des grandes rivières contrôlées dans une enveloppe globale potentielle de $800 \mathrm{~m}^{3} / \mathrm{s}$ et parallèlement d'assurer un débit moyen de vidange de $70 \mathrm{~m}^{3} / \mathrm{s}$ en soutien d'étiage.

A l'achèvement du lac "Aube", s'est posée la question de l'amélioration de la gestion de l'ensemble des ouvrages réalisés dans le domaine des crues. Pour ce faire, une bonne connaissance des crues et de leur évolution sur le bassin était nécessaire, ce qui a conduit l'Institution à utiliser des modèles de simulation d'écoulement existants et notamment le Modèle Seine.

\section{IV $\square$ LE MODÈLE SEINE BARRAGES - RESERVOIRS (MSBR)}

Le Modèle Seine Barrages-Réservoirs est dérivé du Modèle Seine conçu par HYDRATEC (1982/1984) dans le cadre de l'étude de la protection contre les eaux des rivières en région Ile de France.

\subsection{Le Modèle Seine}

Il s'agit d'un modèle de simulation d'écoulement en temps de crue. La zone géographique modélisée correspond au bassin de la Seine en amont de Paris soit la petite Seine depuis la confluence de l'Aube, l'Yonne depuis la confluence de la

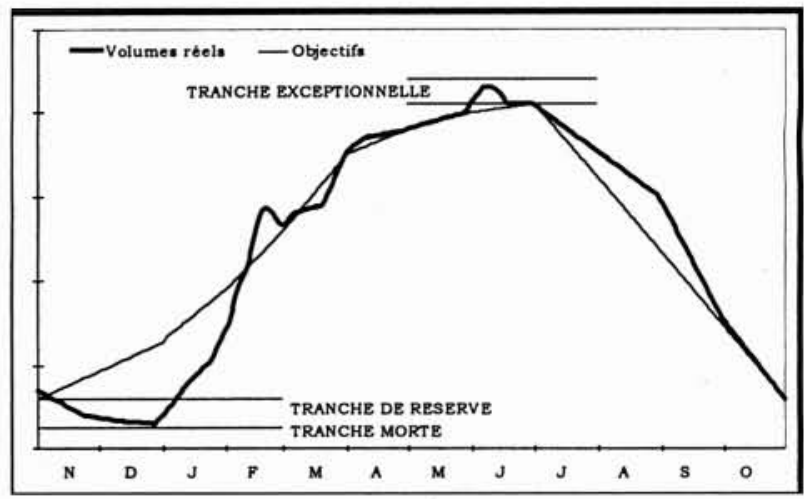

2. Courbe théorique d'exploitation.

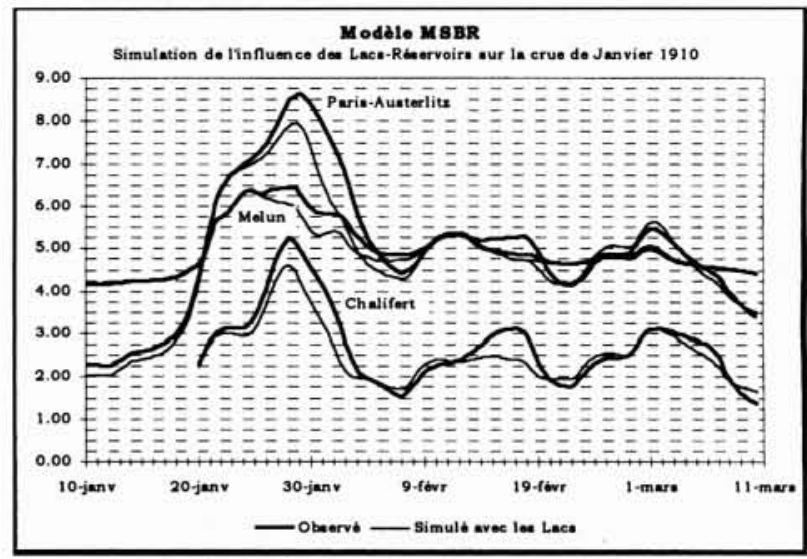

3. Modèle MSBR - Simulation de l'influence des lacs-réservoirs sur la crue de janvier 1910.

Cure, la Marne depuis la confluence de la Saulx et la Seine de Montereau à Paris-Austerlitz soit $625 \mathrm{~km}$ de rivières.

La schématisation de l'écoulement en vallée utilise un découpage en biefs de 1 à $2 \mathrm{~km}$ de longueur tenant compte des singularités de la vallée et représentant ainsi environ 500 nœuds de calcul. Le modèle est renseigné à partir de 60 points d'apport représentant la centaine de petits rus et d'affluents. 4 crues récentes, 1955, 1970, 1978 et janvier 1982, ont été retenues pour caler le modèle aux principales stations et échelles du bassin ( 25 environ).

Le modèle Seine avait pour but de permettre la compréhension des phénomènes dynamiques de propagation des crues en mettant en évidence, pour les crues importantes, les échanges lit majeur - lit mineur, le poids des différents bassins dans la constitution des crues afin de mesurer :

- l'impact hydraulique des aménagements existants et futurs,

- l'importance et l'intérêt des champs d'inondation naturels, - le degré de protection de la région Ile de France suivant les aménagements existants et futurs.

\subsection{Le Modèle Seine Barrages-Réservoirs (MSBR)}

En 1991, à l'initiative de l'Institution, le Modèle Seine a évolué vers une utilisation plus rapide et plus accessible, afin d'avoir la connaissance en temps réel de l'effet des lacs-réservoirs et du déroulement des crues. Les caractéristiques du modèle MSBR ainsi conçu sont :

- la transposition de l'outil informatique sur PC, sous sys- 


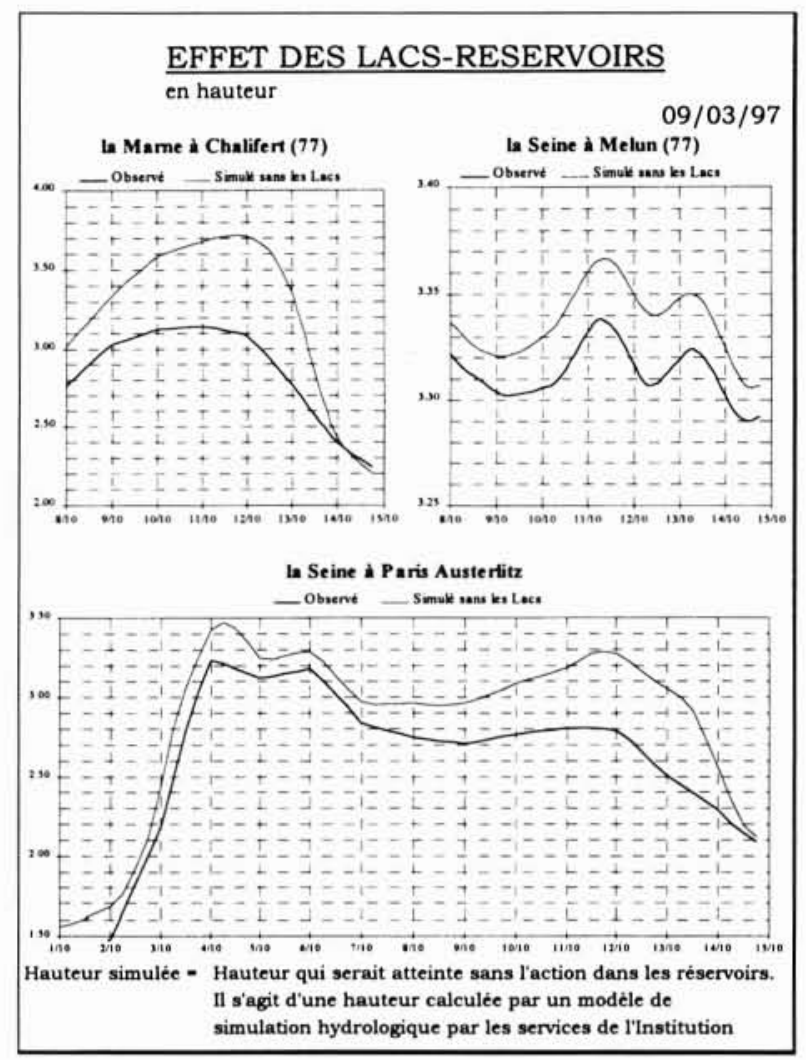

4. Effet des lacs-réservoirs.

tème d'exploitation Windows et une interface d'automatisation des tâches avec les bases de données graphiques et d'élaboration de graphiques spécifiques par menus interactifs,

- un complément de la modélisation en amont de la Seine, de l'Aube et de la Marne pour débuter les simulations en amont des grands lacs,

- l'adjonction de modules de gestion des lacs "Seine", "Aube", "Marne", Pannecière et d'un aménagement sur la Saulx,

- l'utilisation d'un nombre limité de stations hydrométriques, grâce au calage réalisé avec le Modèle Seine, dans le but de favoriser une utilisation en temps réel. 26 stations dont 5 pour l'utilisation des modules de gestion des barrages, ont ainsi été retenues parmi les stations hydrométriques télétransmises du réseau de la DIREN et les informations propres à l'Institution,

- une bibliothèque de 12 crues historiques (1910, 1924, 1955, 1970, 1978, janvier 1982, décembre 1982, 1983, 1991, 1993 et 1995),

- la transposition des débits en hauteurs au droit des 7 stations amont de l'annonce des crues de la région lle de France.

Le domaine d'utilisation de MSBR comprend:

- la simulation des crues historiques avec possibilité d'évaluation de l'effet de la gestion des ouvrages existants ou futurs (module Saulx,...) sur les crues et de variation des paramètres de gestion (règlements d'eau) pour tester les améliorations possibles (fig 3) :

- la constitution de nouvelles hydrologies pour compléter la bibliothèque de crues et l'enrichir des crues dès leur apparition, - l'évaluation en temps réel de l'action des réservoirs sur la crue (fig 4),

- la prévision de l'évolution d'une crue en cours sur 1 jour- née, avec une fourchette de tendance à 2 ou 3 jours suivant les hypothèses pluviométriques de Météo France, en intégrant l'effet des lacs (fig 5).

\section{BILAN DE L'ACTION DES LACS}

Le rôle écrêteur de crues des lacs-réservoirs existants ne peut être cependant que partiel pour des raisons de choix du type d'aménagement structurel et des limites des possibilités de gestion.

Toutefois, les ouvrages réalisés apportent une contribution reconnue à la lutte contre les inondations tout le long des rivières concernées et au soutien en étiage des débits des rivières contrôlées, comme on a pu le constater de 1989 à 1993, tant du point de vue quantitatif que qualitatif.

\section{- 5.1 Rôle écrêteur limité par la stratégie retenue pour l'aménagement du bassin}

L'équipement du bassin en réservoirs résulte d'un choix d'ouvrage à buts multiples qui obligent une gestion partagée entre ces différentes fins et notamment le remplissage des ouvrages pour le début de l'été.

Les grands réservoirs ne peuvent être implantés que dans des zones imperméables et non urbanisées donc éloignées de l'agglomération parisienne. Les lacs existants, en amont des bassins, ne contrôlent ainsi que $17 \%$ du bassin de la Seine en amont de Paris.

Le bassin de l'Yonne, affluent rapide et fougueux, qui

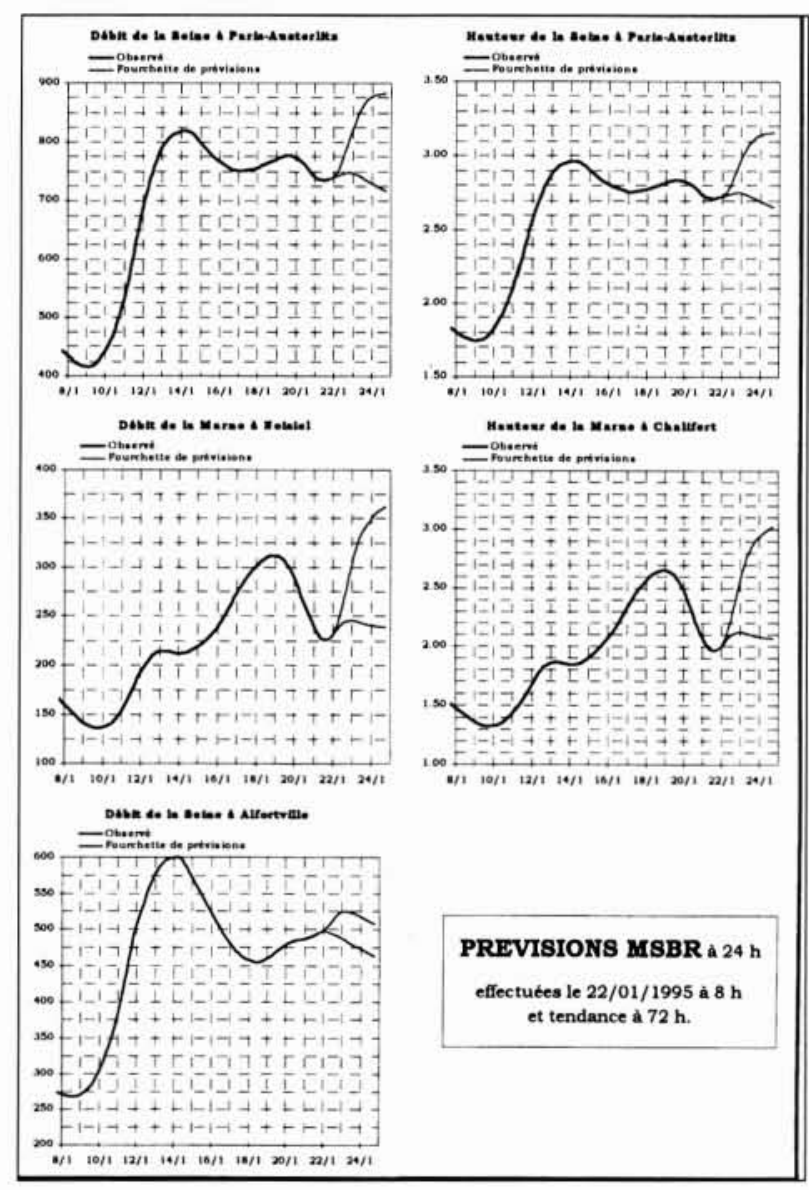

5. Prévisions de crues aux stations de la région parisienne. 
apporte un effet important sur les pointes de crues, est souséquipé, le barrage de Pannecière ne contrôlant que $2 \%$ du bassin versant de cet affluent.

Le bassin intermédiaire, constitué des bassins des affluents secondaires de la Seine et de la Marne (Loing, Essonne, Yerres, Grand Morin, Petit Morin, ... ), qui représente $35 \%$ du bassin versant de la Seine à Paris, ne peut pas être équipé d'ouvrage compte tenu de sa géologie et de son urbanisation.

En 1910, l'accumulation dans les vallées en amont de Paris a dépassé $1000 \mathrm{Mm}^{3}$ pendant qu'un volume de $3000 \mathrm{Mm}^{3}$ s'écoulait à Paris-Austerlitz entre le 18 janvier et le 7 février. Ces valeurs sont à rapprocher de la capacité des ouvrages $\left(830 \mathrm{Mm}^{3}\right)$ et des projets de 1920 qui prévoyaient un volume de stockage de $2400 \mathrm{Mm}^{3}$ pour rabaisser de $5 \mathrm{~m}$ la ligne d'eau de la crue de 1910.

Ces différents points apportent une restriction naturelle à l'efficacité des retenues.

\subsection{Effet de la nature de la crue sur la gestion des ouvrages}

Les prélèvements opérés par les réservoirs se traduisent par une limitation des débits, donc des hauteurs d'eau, à l'aval des ouvrages et plus particulièrement à leur proximité. Au fur et à mesure que l'on s'éloigne des ouvrages, les apports des différents affluents grossissent progressivement le flot principal et tendent à recréer une onde de crue qui se propage progressivement vers l'aval masquant l'effet des prélèvements effectués.

Les grandes crues se classent en différents types :

- crue simple issue d'un épisode pluvieux couvrant l'ensemble du bassin mais isolé dans le temps (1955),

- crue multiple correspondant à 2 épisodes successifs dont le premier couvre l'ensemble du bassin et le second plus faible vient renforcer le premier $(1910,1978)$,

- crue multiple générée par une succession de pluies répétées s'achevant par un événement plus important sur le bassin de l'Yonne (1982).

Cette diversité rend plus difficile l'adaptation de la gestion des ouvrages à l'événement avec un risque de saturer trop vite les réservoirs face aux crues exceptionnelles.

L'influence des réservoirs dépend donc de la nature de la crue, de l'importance respective de l'apport des bassins entre eux et notamment de l'importance des apports du bassin contrôlé par rapport à ceux du bassin intermédiaire non contrôlé.

De ce fait, il est impossible de prévoir comment les ondes de crue des différents bassins se combineront, pendant les 8 à 10 jours de propagation de l'onde entre les réservoirs et Paris, et d'optimiser les prélèvements au droit des réservoirs.

\subsection{Efficacité des lacs-réservoirs}

Les simulations ont permis de quantifier l'action des lacs existants sur les crues historiques. Le tableau 1 résume cette évaluation qui montre que les gains sur la hauteur maximale (H1) sont moindres que les gains maximaux (H2) mais met en évidence l'effet sur la durée de l'inondation par accélération de la décrue.

Parallèlement, dans le créneau d'action des réservoirs qui résulte de la configuration topographique du bassin et de l'effet cumulatif de la réduction de la hauteur d'eau et de la présence de protections locales, il faut avoir à l'esprit l'effet exponentiel du coût des inondations valorisant chaque centimètre gagné pour les grandes crues.

\section{- 5.4 Conclusion}

La région parisienne reste vulnérable aux crues exceptionnelles des différents cours d'eau. Les annales relatent des inondations de forte importance telles que 1658, 1740, 1910 et 1924 montrant la probabilité de retour de tels événements.

L'action d'écrêtement des crues par les réservoirs existants apparaît limitée car, pour maîtriser totalement les crues, il faudrait pouvoir contrôler l'écoulement sur une grande partie des sous-bassins et donc disposer de réservoirs de capacités suffisantes, répartis sur l'ensemble du bassin, ce qui ne paraît pas réalisable.

Toutefois, l'efficacité économique est beaucoup plus importante que l'efficacité hydraulique compte tenu des caractéristiques topographiques et urbanistiques du bassin. Une meilleure protection est à attendre des outils de simulation permettant de développer l'information, la prévision et l'aide à la programmation de nouveaux ouvrages dont l'action doit se conjuguer avec un programme de protections locales. L'aménagement du bassin pour le volet crue nécessite ces connaissances pour se fixer des objectifs de protection qui devront être étayés par la réalisation d'études économiques.

\begin{tabular}{|c|c|c|c|c|}
\hline CRUES & 1982 & 1978 & 1955 & 1910 \\
\hline type de crue & multiple & multiple double & simple & multiple double \\
\hline H1 & $0,10 \mathrm{~m}$ & $0,25 \mathrm{~m}$ & $0,80 \mathrm{~m}$ & $0,90 \mathrm{~m}$ \\
\hline $\mathrm{H} 2$ & $0,55 \mathrm{~m}$ & $0,30 \mathrm{~m}$ & $1,20 \mathrm{~m}$ & $1,50 \mathrm{~m}$ \\
\hline
\end{tabular}

Tableau 1. - Types de crues et gains à l'échelle d'Austerlitz. 